Original Research Paper

\title{
Extraction Optimization of Essential Oil from Medicago hispida and Preliminary Inhibitory Mechanism Against Escherichia coli
}

\author{
Xiulan Chu, Qianfeng Chen, Tong Wang, Xinyu Bai, Chen Pan, Yang Zhang and Lixue Zheng \\ School of Biology and Food Engineering, Changshu Institute of Technology, Changshu, Jiangsu 215500, China
}

\author{
Article history \\ Received: 12-08-2021 \\ Revised: 10-11-2021 \\ Accepted: 03-11-2021 \\ Corresponding Author: \\ Yang Zhang \\ School of Biology and Food \\ Engineering, Changshu \\ Institute of Technology, \\ Changshu, Jiangsu 215500, \\ China
}

Email: zhangyang@cslg.edu.cn

Lixue Zheng

School of Biology and Food

Engineering, Changshu

Institute of Technology,

Changshu, Jiangsu 215500,

China

Email: zhenglixue@cslg.edu.cn

\begin{abstract}
In this study, the extraction optimization, characterization, antioxidant evaluation and preliminary inhibitory mechanism against Escherichia coli of the essential oil from dried Medicago hispida (EOMH) were explored to uncover the pending questions that had been raised in our previous work and to enlarge the industrial potential of EOMH production. The optimal parameters for EOMH extraction were: Extraction time of $7 \mathrm{~h}$, particle size of 20 mesh and liquid-tosolid ratio of 30: $1(\mathrm{~mL} / \mathrm{g})$, under which the highest yield of EOMH reached $0.31 \%$. Nineteen constituents were identified in EOMH by gas chromatography mass spectrometry analysis, among which phytol content was up to $53.6 \%$. These structures were further confirmed by infrared spectroscopy analysis. EOMH exerted similar scavenging capacities against Diphenyl Picryl Hydrazinyl (DPPH) and hydroxyl radicals with those of phytol. However, EOMH elicited stronger inhibitory effect on $E$. coli than that of phytol via shrinking $E$. coli cells, indicating that other factors, in addition to the antioxidant mechanism would equally affect the inhibitory effect of EOMH against E. coli, which should be deepened in the near further. In summary, present work would provide evidence for the industrial production and application of EOMH.
\end{abstract}

Keywords: Medicago hispida, Essential oil, Extraction Optimization, Phytol, Antioxidation, Inhibition on E. coli

\section{Introduction}

Essential Oils (EOs) of plants are volatile aromatic substances extracted and refined from flowers, leaves, roots, seeds or fruits and are existed mostly as colorless or pale yellow oily viscous liquids at room temperature (Mirza and Navaei, 2009). EOs are almost insoluble in water, but are highly soluble in low-polarity organic solvents, including ether and petroleum ether. Meanwhile, EOs are complex mixtures composed of diverse compounds. Generally, one type of EOs contains tens to hundreds of components (Mirza and Navaei, 2009). According to chemical structures, EOs can be divided into four types, including terpenes, aromatics, aliphatics and sulfur/nitrogencontaining compounds. Special compositions endow the EOs with various bioactivities (Huang et al., 2012; Wang et al., 2017), such as antioxidant, antibacterial and antiviral activities. So far, the methods for EOs extraction are focused on steam distillation (Cassel et al., 2009), organic solvent extraction, ultrasonic wave and/or microwave-assisted extractions (Guo et al., 2012). Although advanced auxiliary extraction techniques exhibit certain advantages on increasing extraction yield, traditional steam distillation is still the most frequently considered method for EOs extraction due to low cost and easier operation.

Current researches on genus Medicago are concentrated on Medicago sativa. For instance, comprehensively evaluated the quality of $M$. sativa using near-infrared spectroscopy (Wang et al., 2016). Studied the bioactivities of M. sativa (Liu et al., 2018). Other researchers (Krakowska et al., 2017) were focused on the structureactivity relationships of active substances extracted and purified from $M$. sativa. In summary, $M$. sativa is the species belonging to Medicago family that has been received comprehensively studied and the substances of interest are polysaccharides, flavonoids and saponins. However, there is less research or exploitation on Medicago hispida, another common species of Medicago. In China, M. hispida is usually consumed as delicious vegetable and it contains many nutrients such as carbohydrates, proteins, vitamins and trace elements. The medicinal use of M. hispida was first described in Mingyi Bielu in the Han dynasty. Modern pharmacological investigations confirmed the hypoglycemic effects of total saponins from $M$. hispida and the antibacterial and antioxidant activities of total flavonoids from M. hispida (Cheng et al., 2016). Nevertheless, there is little information 
on the EOs from M. hispida. In previous work (Zheng et al., 2019), we extracted the essential oil from fresh $M$. hispida (EOFMH) by using stem distillation and analyzed the chemical components with gas Chromatography-Mass Spectrometry (GC-MS). At the same time, we found that EOFMH exerts significant inhibitory activity against $E$. coli, which is similar to that of Chloromycetin, a chemically obtained antibacterial drug and EOFMH is dominated by phytol, accounting for $48.8 \%$ of the total content of EOs in EOFMH. Thus we conjectured that the promising inhibitory activity against $E$. coli of EOFMH would be mainly contributed by the abundant phytol and the bacteriostatic activity would be partially related to the antioxidant potential contributed by phytol. However, these hypothesizes are urgently needed to be confirmed by further experimental studies.

In the present investigation, considering the promising inhibitory activity against $E$. coli, firstly, we further optimized the steam distillation process of essential oil from M. hispida (EOMH) via Response Surface Methodology (RSM) coupled with Box-Behnken Design (BBD) involving liquid-solid ratio, extraction time and particle size as single factors. Meanwhile, due to the fact that dried raw material is easier to be preserved, especially for continuous industrial production, in this study, dried $M$. hispida was selected as raw and processed materials instead of fresh ones. Secondly, GC-MS and Fourier Transform Infrared spectroscopy (FT-IR) were used to identify and confirm the chemical profile of obtained EOMH. Thirdly, the in vitro antioxidant capacities of EOMH were tested. Finally, the preliminary mechanism of EOMH against $E$. coli was uncovered by Scanning Electron Microscopy (SEM). This continuous work is more likely to expand and validate our previous work (Zheng et al., 2019) and to underlie the further researches on EOMH theoretically.

\section{Materials and Methods}

\section{Materials and Chemicals}

M. hispida materials used in this study were collected at the farmland of Southeast Development Zone, Changshu, Jiangsu, China on November 11, 2020 and were authenticated by Prof. Jianhua Hao, Changshu Institute of Technology, Changshu, Jiangsu, China. All the reagents and chemicals were analytically pure and microbial strain was stored at School of Biology and Food Engineering, Changshu Institute of Technology, Changshu, Jiangsu, China.

\section{Essential Oil Extraction}

Fresh and cleaned M. hispida were dried at $60^{\circ} \mathrm{C}$ in an electric blast drying oven, crushed and sieved into different particle sizes. At each time, an appropriate amount of the crushed samples was put into a $2000 \mathrm{~mL}$ flask and then added certain water for soaking overnight. The flask was connected to a steam distilling apparatus for certain time of reflux extraction at $105^{\circ} \mathrm{C}$. After heating and cooling down, the liquids at the bottom of the flask were filtered out and the upper yellow oil was the obtained Essential oil from $M$. hispida (EOMH), which was collected and reserved in a collecting tube for further analysis (Cui et al., 2018).

The essential oil extraction rate $Q(\%)$ was calculated as follows:

$Q=\frac{m}{M} \times 100 \%$

Where:

$m$ : Mass of essential oil, $\mathrm{g}$

M: Mass of dried M. hispida, $\mathrm{g}$

\section{Single-Factor Tests}

Single-factor tests involving three factors (extraction time, particle size, liquid-to-solid ratio) were conducted. The effects of different factors on EOMH extraction rate were studied. The three factors were set as follows: extraction time $(3 \sim 11 \mathrm{~h})$, particle size $(0 \sim 40 \mathrm{mesh})$ and liquid-to-solid ratio (10: $1 \sim 50: 1 \mathrm{~mL} / \mathrm{g}$ ) (Cui et al., 2018).

\section{Response Surface Methodology (RSM) Optimization}

According to the results of single-factor tests, the effects of process parameters on EOMH extraction rate were optimized by RSM coupled with Box-Behnken Design (BBD). The effects of different factors, either alone or in combination on EOMH extraction rate were compared to optimize the EOMH extraction. The factor levels and codes of BBD were listed in Table 1.

The experimental data were analyzed by multifactor fitting regression on Design-Expert 8.0.6.1. Then a two-order polynomial model in the form of equations was built:

$$
Y=\beta_{0}+\sum_{i=1}^{n} \beta_{i} X_{i}+\sum_{i=1}^{n} \beta_{i i} X_{i}^{2}+\sum_{i<j}^{n} \beta_{i j} X_{i} X_{j}+\varepsilon
$$

Where:

$Y=$ The predicted extraction rate of EOMH

$\beta_{0} \quad=$ A constant coefficient

$\beta_{i}, \beta_{i i}$ and $\beta_{i j}=$ First-order quadratic coefficients of $X_{i}$

$\varepsilon \quad=$ The experimental error

Table1: The factor levels and codes used in the RSM

\begin{tabular}{llll}
\hline & Level & \\
& --------------- \\
\hline & -1 & 0 & 1 \\
$X_{1}:$ Extraction time (h) & 5 & 7 & 9 \\
$X_{2}:$ Particle size (mesh) & 10 & 20 & 30 \\
$X_{3}$ : Liquid-to-solid ratio (mL/g) & $20: 1$ & $30: 1$ & $40: 1$ \\
\hline
\end{tabular}




\section{GC-MS Analysis}

Chemical constituents of EOMH were analyzed by a QP-2010 gas chromatography mass spectrometry (GC-MS) (Shimadzu, Kyoto, Japan) installed with a Rxi-5Sil MS column $(30 \mathrm{~m} \times 0.25 \mathrm{~mm} \times 0.25 \mu \mathrm{m})$. The analytical processes were as follows: Helium was served as carrier gas with flow rate of $1.0 \mathrm{~mL} / \mathrm{min}$; the initial column temperature was set to $40^{\circ} \mathrm{C}$ (maintained for $4 \mathrm{~min}$ ), then reached to $60^{\circ} \mathrm{C}$ (heating rate of $5^{\circ} \mathrm{C} / \mathrm{min}$, maintained 2 min at $60^{\circ} \mathrm{C}$ ), raised from $60^{\circ} \mathrm{C}$ to $110^{\circ} \mathrm{C}$ at $15^{\circ} \mathrm{C} / \mathrm{min}$ (maintained $4 \mathrm{~min}$ at $110^{\circ} \mathrm{C}$ ), then raised from $110^{\circ} \mathrm{C}$ to $180^{\circ} \mathrm{C}$ at $5^{\circ} \mathrm{C} / \mathrm{min}$ (maintained $5 \mathrm{~min}$ at $180^{\circ} \mathrm{C}$ ), finally climbed to $280^{\circ} \mathrm{C}$ at $25^{\circ} \mathrm{C} / \mathrm{min}$ (maintained $5 \mathrm{~min}$ at $280^{\circ} \mathrm{C}$ ); ion trap temperature was $230^{\circ} \mathrm{C}$; transmission line temperature was $250^{\circ} \mathrm{C}$; scan range was from 35 to $500 \mathrm{amu}$ and ionization energy was $70 \mathrm{eV}$. One microliter of EOMH was dissolved in ether to a total concentration of $12 \mathrm{mg} / \mathrm{mL}$ and injected into the column at a split ratio of 1: 30. Constituents in EOMH were authenticated though comparing both the Retention Index (RI) relative to the mass spectra library (NIST 05) (Elkady and Ayoub, 2018) and the standards of $\mathrm{C}_{8}-\mathrm{C}_{40} n$-alkanes.

\section{FT-IR Analysis}

Based on a method from Boughendjioua et al. (2020), EOMH and phytol standard were characterized by FT-IR. At each time, $50 \mu \mathrm{L}$ of sample was dripped on a glass slide, which was gently shaken to make the sample distribute evenly to form a thin layer. After acquisition of background information, the sample was scanned by using a Fourier transform infrared spectrometer from 4000 to $400 \mathrm{~cm}^{-1}$.

\section{The In Vitro Antioxidant Activities}

\section{DPPH Radical Scavenging Capability}

The method from Javad et al. 2009 was adopted with minor modifications. EOMH and phytol standard were both dissolved in dimethyl sulfoxide to produce the final concentrations of $0.2,0.4,0.6,0.8,1.0,1.2 \mathrm{mg} / \mathrm{mL}$. Each sample $(2 \mathrm{~mL})$ was sucked into a test tube and mixed with $1 \mathrm{~mL}$ of $0.1 \mathrm{mmol} / \mathrm{L}$ DPPH solution, which was tested sample. The control sample was prepared by using anhydrous ethanol instead of the DPPH solution. The blank sample was prepared by using anhydrous ethanol instead of tested sample. The three groups of samples were completely oscillated and evenly mixed, followed by being placed in the dark for $30 \mathrm{~min}$. After that, the absorbance at $517 \mathrm{~nm}$ of each sample was detected. Vitamin $\mathrm{C}(\mathrm{Vc})$ was used as positive control, each test was repeated for three times. DPPH radical scavenging rate was calculated as follows:

$D P P H$ radical scavenging rate $(\%)=\left(1-\frac{A_{\mathrm{i}}-A_{\mathrm{j}}}{A_{0}}\right) \times 100$
Where:

$A_{0}$ : Absorbance of the blank

$A_{i}$ : Absorbance of the sample

$A_{j}$ : Absorbance of the control

\section{Hydroxyl Radical Scavenging Capability}

The method from Chen et al., 2007 was referenced with minor modifications. EOMH and phytol standard were dissolved in dimethyl sulfoxide to give the final concentrations of $0.2,0.4,0.6,0.8,1.0,1.2 \mathrm{mg} / \mathrm{mL}$, respectively. Each sample $(1 \mathrm{~mL})$ was sucked into a test tube and mixed with $1 \mathrm{~mL}$ of $6 \mathrm{mmol} / \mathrm{L} \mathrm{FeSO}_{4}$ solution and $1 \mathrm{~mL}$ of $6 \mathrm{mmol} / \mathrm{L} \mathrm{H}_{2} \mathrm{O}_{2}$ solution. The sample was evenly shaken and placed at room temperature for $10 \mathrm{~min}$. Then $1 \mathrm{~mL}$ of $6 \mathrm{mmol} / \mathrm{L}$ salicylic acid solution was added. The blank sample was prepared by replacing tested sample with distilled water. The control sample was obtained by substituting distilled water for salicylic acid solution. The three groups of samples were fully shaken and placed in water bath at $37^{\circ} \mathrm{C}$ for $0.5 \mathrm{~h}$. After that, the absorbance at $510 \mathrm{~nm}$ was detected. Vc was served as positive control. Each sample was tested in triplicate. The hydroxyl radical scavenging rate was computed as follows:

Hydroxyl radical scavenging rate $(\%)=\left(1-\frac{A_{\mathrm{i}}-A_{\mathrm{j}}}{A_{0}}\right) \times 100$

Where:

$A_{0}$ : Absorbance of the blank

$A_{i}$ : Absorbance of the sample

$A_{j}$ : Absorbance of the control

\section{Preliminary Inhibitory Mechanism against E. coli}

The morphological changes of E. coli were scanned by SEM (Zhan et al., 2014a). Three groups were set as follows: One group was treated with EOMH, another group was dealed with phytol and one was the activated E. coli suspension (600 nm, OD: 0.5) (blank control). EOMH and phytol standard were dissolved in dimethyl sulfoxide, respectively. All samples $(20 \mu \mathrm{L}$ of each) were fully mixed with $180 \mu \mathrm{L}$ of above-mentioned suspension, forming the treated groups. Then $200 \mu \mathrm{L}$ of suspension was used as the blank control. After cultivation in a thermostatic incubator at $37^{\circ} \mathrm{C}$ for $16 \mathrm{~h}$, one drop from each sample was collected and put into a dissociated mica slide, which was metal-sprayed and observed under SEM.

\section{Statistical Analysis}

All the assays in this study were performed in triplicate and the results were presented as means or means \pm standard error. The experimental data were statistically tested by analysis of one-way Analysis of Variance (ANOVA). $P<0.05$ was deemed as significant difference. 


\section{Results}

\section{Single-Factor Tests}

\section{Effects of Extraction Time, Particle Size and Liquid-to- Solid Ratio on Extraction Rate of EOMH}

The effect of extraction time on EOMH extraction rate was illustrated in Fig. 1, which showed that the optimal extraction time was 7 h. As shown in Fig. 2, the extraction rate of EOMH was maximized when the particle size of dried M. hispida reached 20 mesh. The effect of liquid-to-solid ratio on EOMH extraction rate was illustrated in Fig. 3 and the extraction rate was maximized to $0.25 \%$ at the liquid-tosolid ratio of $30: 1 \mathrm{~mL} / \mathrm{g}$. The effects of above-mentioned three single factors on the extraction rate of EOMH all appeared an upward trend first and then a downward trend.

\section{RSM Optimization}

The extraction rate of EOMH was optimized by RSM. Experimental data in Table 2 were fitted on Design-Expert software. A regression equation was established as follows:

$$
\begin{aligned}
& Y=-1.33794+0.19538 X_{1}+0.042275 X_{2}+0.036475 X_{3} \\
& -1.125 \times 10^{-3} X_{1} X_{2}+1.25 \times 10^{-4} X_{1} X_{3}+7.5 \times 10^{-5} X_{2} X_{3} \\
& -0.012438 X_{1}^{2}-9.475 \times 10^{-4} X_{2}^{2}-6.475 \times 10^{-4} X_{3}^{2}
\end{aligned}
$$

The calibration coefficient was $\mathrm{R}^{2}=0.9823$ and the Coefficient of Variation (CV) was $6.58 \%$. Simulation and fitting with regression equations showed that this experimental model can well reflect the relationships of different factors with EOMH extraction rate and could be effective for simulating computation and thereby returning real experimental data.

The model was featured with $P<0.0001$ and error $P=$ 0.3717 (Table 3). Analysis of Variance (ANOVA) and tests proved that this model is highly significant and can yield accurate data. Results of ANOVA showed that among the single terms, the influence of particle size $\left(X_{2}\right)$ on EOMH yield surpasses those of extraction time $\left(X_{I}\right)$ and liquid-to-solid ratio $\left(X_{3}\right)$. The influence of betweenfactor interaction on extraction rate ranked as: $X_{1} X_{2}>X_{2} X_{3}$ $>X_{1} X_{3}$. The effects of squares of each factor on the extraction rate ranked as: $X_{2}^{2}>X_{3}^{2}>X_{l}^{2}$.

During the extraction of EOMH, the contours and 3D RSM images illustrated the effects of between-factor interactions on the extraction rate (Fig. 4). As for the interaction between extraction time and particle size, the RSM images (Fig. 4b) were abrupt and the contours were ellipse-shaped (Fig. 4a). The interaction between extraction time and particle size significantly affected EOMH yield $(P$ $=0.015$ ) (Huang et al., 2013). As for the interaction between extraction time and liquid-to-solid ratio, the contours in Fig. $4 \mathrm{c}$ were shaped similarly as that in Fig. 4e, but the 3D RSM images were more circular. Hence, the influence of particle size on EOMH extraction slightly surpassed the influence of liquid-to-solid ratio. As for the interaction between liquid-tosolid ratio and particle size, the RSM images (Fig. 4f) were gentle and the contours (Fig. 4e) were nearly round-shaped. Therefore, the interaction between liquid-to-solid ratio and particle size did not significantly affect EOMH extraction rate $(P=0.3208)$. The results from the contours and 3D RSM images were generally

\begin{tabular}{|c|c|c|c|c|c|}
\hline \multicolumn{4}{|c|}{ Independent variable } & \multicolumn{2}{|c|}{ Extraction rate $(\%)$} \\
\hline Run & $X_{1}$ & $X_{2}$ & $X_{3}$ & Actual value & Predicted value \\
\hline$\overline{1}$ & -1 & -1 & 0 & 0.15 & 0.15 \\
\hline 2 & 1 & -1 & 0 & 0.22 & 0.21 \\
\hline 3 & -1 & 1 & 0 & 0.16 & 0.17 \\
\hline 4 & 1 & 1 & 0 & 0.14 & 0.14 \\
\hline 5 & -1 & 0 & -1 & 0.20 & 0.20 \\
\hline 6 & 1 & 0 & -1 & 0.19 & 0.20 \\
\hline 7 & -1 & 0 & 1 & 0.20 & 0.19 \\
\hline 8 & 1 & 0 & 1 & 0.20 & 0.21 \\
\hline 9 & 0 & -1 & -1 & 0.17 & 0.17 \\
\hline 10 & 0 & 1 & -1 & 0.14 & 0.13 \\
\hline 11 & 0 & -1 & 1 & 0.15 & 0.16 \\
\hline 12 & 0 & 1 & 1 & 0.15 & 0.15 \\
\hline 13 & 0 & 0 & 0 & 0.32 & 0.31 \\
\hline 14 & 0 & 0 & 0 & 0.31 & 0.31 \\
\hline 15 & 0 & 0 & 0 & 0.30 & 0.31 \\
\hline 16 & 0 & 0 & 0 & 0.33 & 0.31 \\
\hline 17 & 0 & 0 & 0 & 0.30 & 0.31 \\
\hline
\end{tabular}
consistent with the results presented in Table 3.

Table 2: Box-Behnken design and experimental data

$X_{1}:$ Extraction time $(\mathrm{h})$

$X_{2}$ : Particle size (mesh);

$X_{3}$ : Liquid-to-solid ratio $(\mathrm{mL} / \mathrm{g})$ 
Table 3: Analysis of variance

\begin{tabular}{|c|c|c|c|c|c|c|}
\hline Source & Df & Sum of squares & Mean square & $F$-value & $P$-value & Significance $^{\mathrm{a}}$ \\
\hline Model & 9 & 0.077 & $8.512 \times 10^{-3}$ & 43.18 & $<0.0001$ & $* *$ \\
\hline$X_{1}$ & 1 & $2.000 \times 10^{-4}$ & $2.000 \times 10^{-4}$ & 1.01 & 0.3474 & n.s. \\
\hline$X_{2}$ & 1 & $1.250 \times 10^{-3}$ & $1.250 \times 10^{-3}$ & 6.34 & 0.0399 & $*$ \\
\hline$X_{3}$ & 1 & 0.000 & 0.000 & 0.000 & 1.0000 & n.s. \\
\hline$X_{1} X_{2}$ & 1 & $2.025 \times 10^{-3}$ & $2.025 \times 10^{-3}$ & 10.27 & 0.0150 & * \\
\hline$X_{1} X_{3}$ & 1 & $2.500 \times 10^{-5}$ & $2.500 \times 10^{-5}$ & 0.13 & 0.7323 & n.s. \\
\hline$X_{2} X_{3}$ & 1 & $2.250 \times 10^{-4}$ & $2.250 \times 10^{-4}$ & 1.14 & 0.3208 & n.s. \\
\hline$X_{1}^{2}$ & 1 & 0.010 & 0.010 & 52.86 & 0.0002 & $* *$ \\
\hline$X_{2}^{2}$ & 1 & 0.038 & 0.038 & 191.74 & $<0.0001$ & $* *$ \\
\hline$X_{3}^{2}$ & 1 & 0.018 & 0.018 & 89.54 & $<0.0001$ & $* *$ \\
\hline Residual & 7 & $1.380 \times 10^{-3}$ & $1.971 \times 10^{-4}$ & & & \\
\hline Lack of fit & 3 & $7.000 \times 10^{-4}$ & $2.333 \times 10^{-4}$ & 1.37 & 0.3717 & n.s. \\
\hline Pure error & 4 & $6.800 \times 10^{-4}$ & $1.700 \times 10^{-4}$ & & & \\
\hline Cortotal & 16 & 0.078 & & & & \\
\hline
\end{tabular}

${ }^{a^{*}} P<0.05$ significant ; ${ }^{* *} P<0.01$ highly significant ; $P>0.05$ not significant (n.s.); Lack of fit means the model is significant

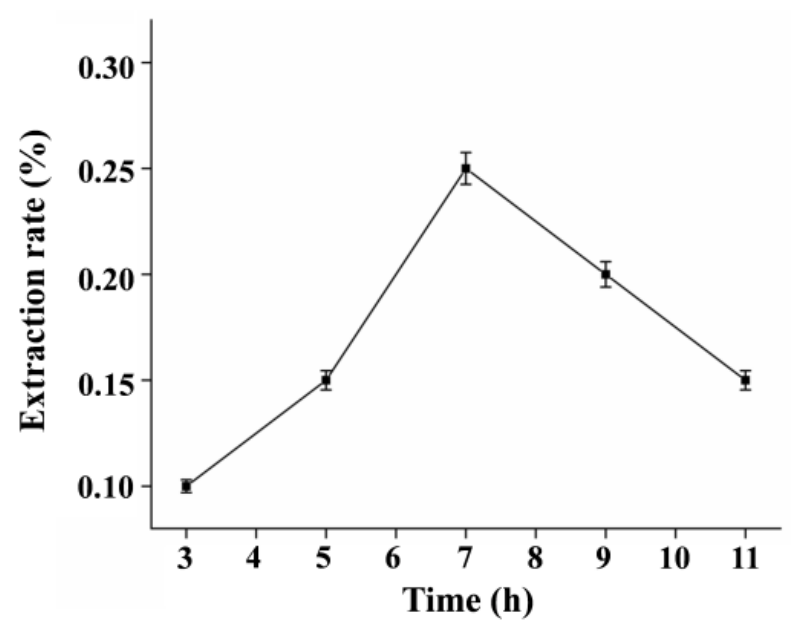

Fig. 1: The effects of extraction time on the extraction rate of EOMH. Data was shown as means \pm SD $(n=3)$

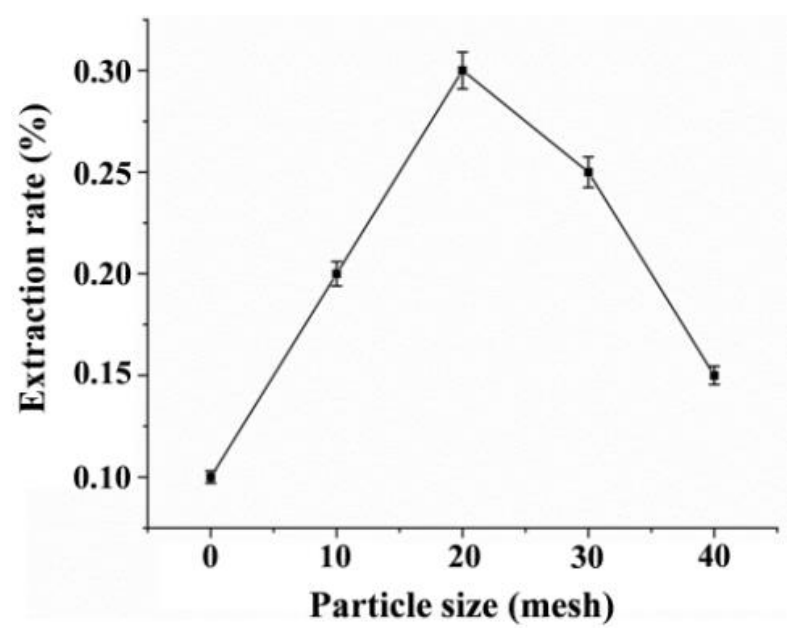

Fig. 2: The effects of particle size on the extraction rate of EOMH. Data was shown as means $\pm \mathrm{SD}(\mathrm{n}=3)$

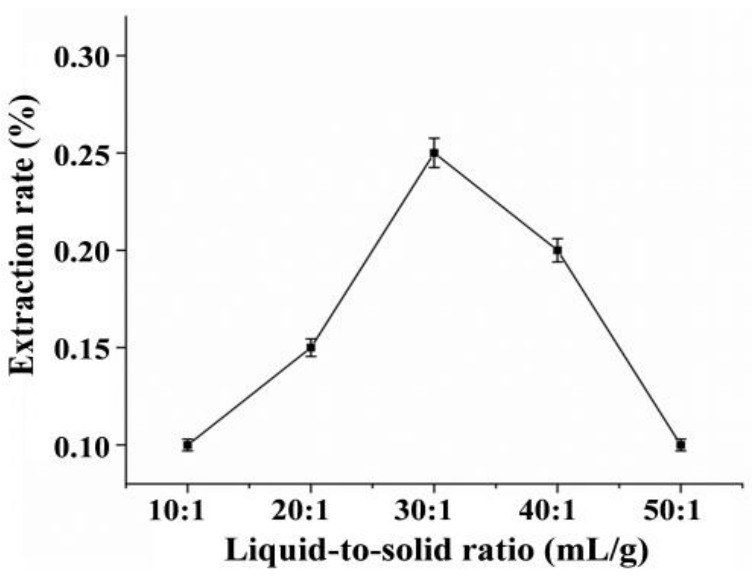

Fig. 3: The effects of liquid-to-solid ratio on the extraction rate of EOMH. Data was shown as means $\pm \operatorname{SD}(n=3)$

\section{Verification of Optimal Process Parameters}

Based on the RSM model, the optimal process for EOMH extraction was: Extraction time of $7.28 \mathrm{~h}$, particle size of 18.49 mesh and liquid-to-solid ratio of 29.94: 1 $(\mathrm{mL} / \mathrm{g})$. To facilitate the practical operation, we adjusted the optimal process into extraction time of $7 \mathrm{~h}$, particle size of 20 mesh and liquid-to-solid ratio of $30: 1(\mathrm{~mL} / \mathrm{g})$. The average extraction rate from three parallel experiments was $0.31 \%$, which was close to the predicted value, indicating that the obtained model is feasible for predicting the optimization of EOMH extraction.

\section{GC-MS Profile}

Nineteen constituents were identified in EOMH by GCMS and results were presented in Table 4. These components were identical with the essential oil extracted from fresh M. hispida, but the content of each compound was different (Zheng et al., 2019). Compared with EOFMH, 
there were eight compounds missing in EOMH, the content of phytol in EOMH was raised to $53.6 \%$, which was $9.84 \%$ higher than that in EOFMH.

\section{FT-IR Analysis}

The chemical profile of EOMH was further confirmed by FT-IR using phytol as control. From Fig. 5, it can be seen that the chemical structure of phytol contains special groups, including one carbon-carbon double bond $(\mathrm{C}=\mathrm{C})$ and one hydroxyl group $(\mathrm{O}-\mathrm{H})$. The FTIR spectrum of EOMH showed peaks at $3433 \mathrm{~cm}^{-1}$ (stretching vibration of $\mathrm{O}-\mathrm{H}$ ) and $1644 \mathrm{~cm}^{-1}$ (stretching vibration of $\mathrm{C}=\mathrm{C}$ ). These peaks were similar to the stretching vibration peaks of standard phytol at 3373 $\mathrm{cm}^{-1}(\mathrm{O}-\mathrm{H})$ and $1659 \mathrm{~cm}^{-1}(\mathrm{C}=\mathrm{C})$. The result of FT-IR analysis was highly consistent with that of GC-MS determination (Table 4).

\section{In Vitro Antioxidant Activity}

\section{DPPH Radical-Scavenging Capacity}

Within the concentration range of $0.2 \mathrm{mg} / \mathrm{mL}$ $1.2 \mathrm{mg} / \mathrm{mL}$, EOMH can scavenge $\mathrm{DPPH}^{*}$ to some extent (Fig. 6a). When the concentration gradually increased, the scavenging capability of EOMH against $\mathrm{DPPH}^{\cdot}$ was generally improved. At the concentration of $1.2 \mathrm{mg} / \mathrm{mL}$, the scavenging rate was up to $65.05 \%$. The $\mathrm{DPPH}^{\circ}$ scavenging capability of phytol changed in the similar way as that of EOMH. At the concentration of $1.2 \mathrm{mg} / \mathrm{mL}$, the scavenging rate reached $70.05 \%$. The half maximal inhibitory concentration $\left(\mathrm{IC}_{50}\right)$ values of $\mathrm{EOMH}$ and phytol were $0.022 \mathrm{mg} / \mathrm{mL}$ and $0.036 \mathrm{mg} / \mathrm{mL}$, respectively. In comparison, the $\mathrm{DPPH}^{*}$ scavenging capability of EOMH was much stronger than that of phytol, but the $\mathrm{DPPH}^{*}$ scavenging abilities were generally weaker than that of $\mathrm{Vc}$.

\section{Hydroxyl Radical-Scavenging Capacity}

The hydroxyl radical $\left({ }^{\circ} \mathrm{OH}\right)$ scavenging rates of EOMH and phytol were calculated. Then a relationship between concentration and scavenging rate was established (Fig. 6b). Clearly, the $\bullet \mathrm{OH}$ scavenging capabilities of phytol and EOMH were elevated with the increment of concentration within the range of 0.2 $\sim 1.2 \mathrm{mg} / \mathrm{mL}$. At the concentration of $1.2 \mathrm{mg} / \mathrm{mL}$, the -OH scavenging rates of EOMH and phytol were $68.02 \%$ and $72.77 \%$, respectively. The corresponding $\mathrm{IC}_{50}$ values were 0.271 and $0.290 \mathrm{mg} / \mathrm{mL}$, respectively, suggesting that the $\mathrm{OH}$ scavenging capability of EOMH was little weaker than that of phytol and also weaker than that of $\mathrm{Vc}$.

\section{Preliminary Inhibitory Mechanism Against E. coli}

The SEM images of untreated E. coli suspension showed that the thalli was morphologically smooth and even on the whole and was complete and plump, with smooth and uninjured surface (Fig. 7a). After addition of phytol (Fig. 7b), the cell morphology was not changed largely, but the cells shrunk to some extent and were less plentiful. After treatment with EOMH, the cell morphology was changed significantly. The cells shrunk on the whole and the surface was rough with numerous folds (Fig. 7c).

Table 4: Chemical profiles of EOMH

\begin{tabular}{|c|c|c|c|c|c|c|c|}
\hline No. & Compounds & Molecular & & & & & \\
\hline formula & $\mathrm{Rt} / \mathrm{min}$ & RI & $\mathrm{RI}^{*}$ & Type & $\%$ & & \\
\hline 1 & Nonadecane & $\mathrm{C}_{19} \mathrm{H}_{44}$ & 35.28 & 1910 & 1910 & Alkane & 0.09 \\
\hline 2 & Eicosane & $\mathrm{C}_{20} \mathrm{H}_{42}$ & 37.79 & 2009 & 2002 & Alkane & 0.11 \\
\hline 3 & Ethyl heptadecanoate & $\mathrm{C}_{19} \mathrm{H}_{38} \mathrm{O}_{2}$ & 38.98 & 2077 & 2086 & Ester & 0.10 \\
\hline 4 & Elaidyl alcohol & $\mathrm{C}_{18} \mathrm{H}_{36} \mathrm{O}$ & 39.28 & 2061 & 2119 & Alcohol & 0.13 \\
\hline 5 & Heneicosane & $\mathrm{C}_{21} \mathrm{H}_{44}$ & 39.42 & 2109 & 2138 & Alkane & 0.18 \\
\hline 6 & Docosane & $\mathrm{C}_{22} \mathrm{H}_{46}$ & 40.00 & 2208 & 2198 & Alkane & 2.81 \\
\hline 7 & Phytol & $\mathrm{C}_{20} \mathrm{H}_{40} \mathrm{O}$ & 40.12 & 2200 & 2210 & Alcohol & 53.6 \\
\hline 8 & $\begin{array}{l}\text { 2-[[2-[(2-Ethylcyclopropyl) } \\
\text { methyl]cyclopropyl]methyl] } \\
\text { cyclopropaneoctanoic acid } \\
\text { methyl ester }\end{array}$ & $\mathrm{C}_{22} \mathrm{H}_{38} \mathrm{O}_{2}$ & 40.45 & 2266 & 2262 & Ester & 0.38 \\
\hline 9 & Ethyl linolenate & $\mathrm{C}_{20} \mathrm{H}_{34} \mathrm{O}_{2}$ & 40.58 & 2201 & 2250 & Ester & 2.05 \\
\hline 10 & Cis-9-tricosene & $\mathrm{C}_{23} \mathrm{H}_{46}$ & 40.89 & 2315 & 2311 & Alkene & 3.39 \\
\hline 11 & Pentacosane & $\mathrm{C}_{25} \mathrm{H}_{52}$ & 42.25 & 2506 & 2508 & Alkane & 3.51 \\
\hline 12 & 9-Hexacosene & $\mathrm{C}_{26} \mathrm{H}_{52}$ & 42.88 & 2614 & 2582 & Alkene & 2.12 \\
\hline 13 & Diisooctyl phthalate & $\mathrm{C}_{24} \mathrm{H}_{38} \mathrm{O}_{4}$ & 43.41 & 2704 & 2668 & Ester & 1.11 \\
\hline 14 & Hexacosane & $\mathrm{C}_{26} \mathrm{H}_{54}$ & 43.79 & 2606 & 2674 & Alkane & 5.09 \\
\hline 15 & 1-Hexacosanol & $\mathrm{C}_{26} \mathrm{H}_{54} \mathrm{O}$ & 44.51 & 2848 & 2780 & Alcohol & 3.28 \\
\hline
\end{tabular}


Table 4: Continue

\begin{tabular}{|c|c|c|c|c|c|c|c|}
\hline 16 & Methyl pentacosanoate & $\mathrm{C}_{26} \mathrm{H}_{52} \mathrm{O}_{2}$ & 45.12 & 2773 & 2832 & Ester & 2.44 \\
\hline 17 & Nonacosane & $\mathrm{C}_{29} \mathrm{H}_{60}$ & 45.99 & 2904 & 2902 & Alkane & 4.10 \\
\hline 18 & Triacontane & $\mathrm{C}_{30} \mathrm{H}_{62}$ & 47.29 & 3003 & 2998 & Alkane & 6.46 \\
\hline \multirow[t]{5}{*}{19} & Dotriacontane & $\mathrm{C}_{32} \mathrm{H}_{66}$ & 50.39 & 3202 & 3162 & Alkane & 4.31 \\
\hline & Total & & & & & & 95.3 \\
\hline & Diterpene alcohol compounds & & & & & & 53.6 \\
\hline & Alkane compounds & & & & & & 26.7 \\
\hline & Others & & & & & & 15.0 \\
\hline
\end{tabular}

RI is the retention index determined by the database NIST 05; RI* is the retention index calculated according to the retention time of $\mathrm{C}_{8}-\mathrm{C}_{40} n$-alkanes
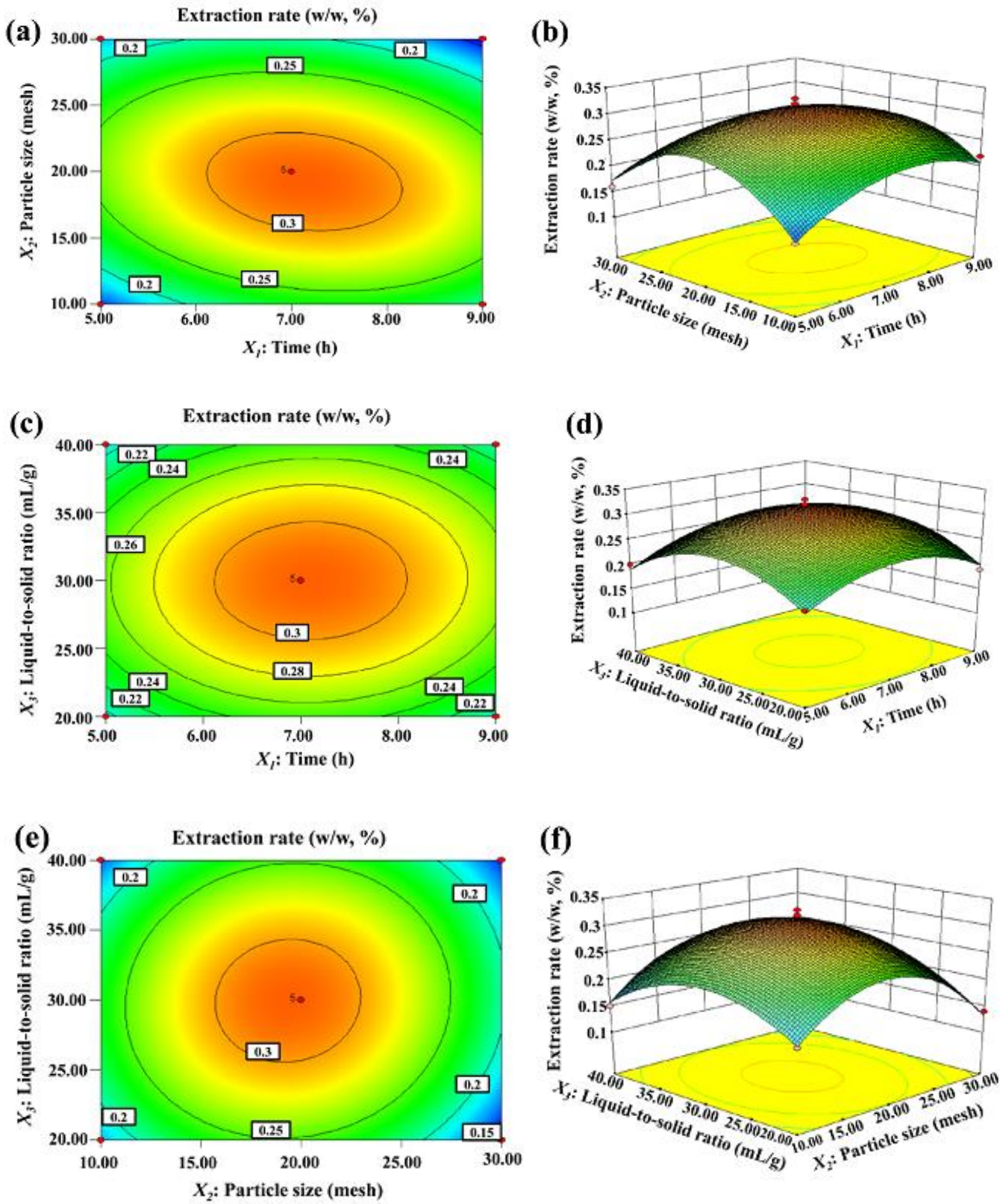

Fig. 4: Contours and 3D RSM images showing the effects of between-factor interactions on the extraction rate. Extraction time versus Particle size (a, b); Extraction time versus Liquid-to-solid ratio (c, d); Particle size versus Liquid-to-solid ratio (e, f) 


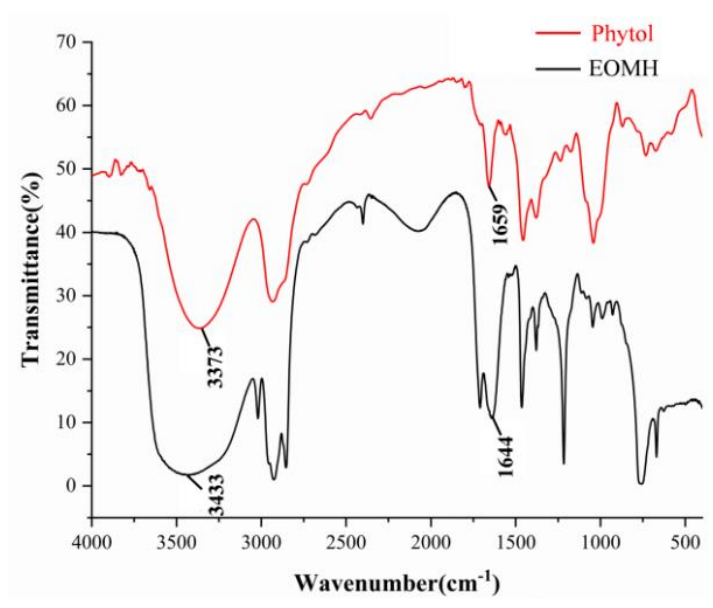

Fig. 5: Infrared spectra of phytol and EOMH
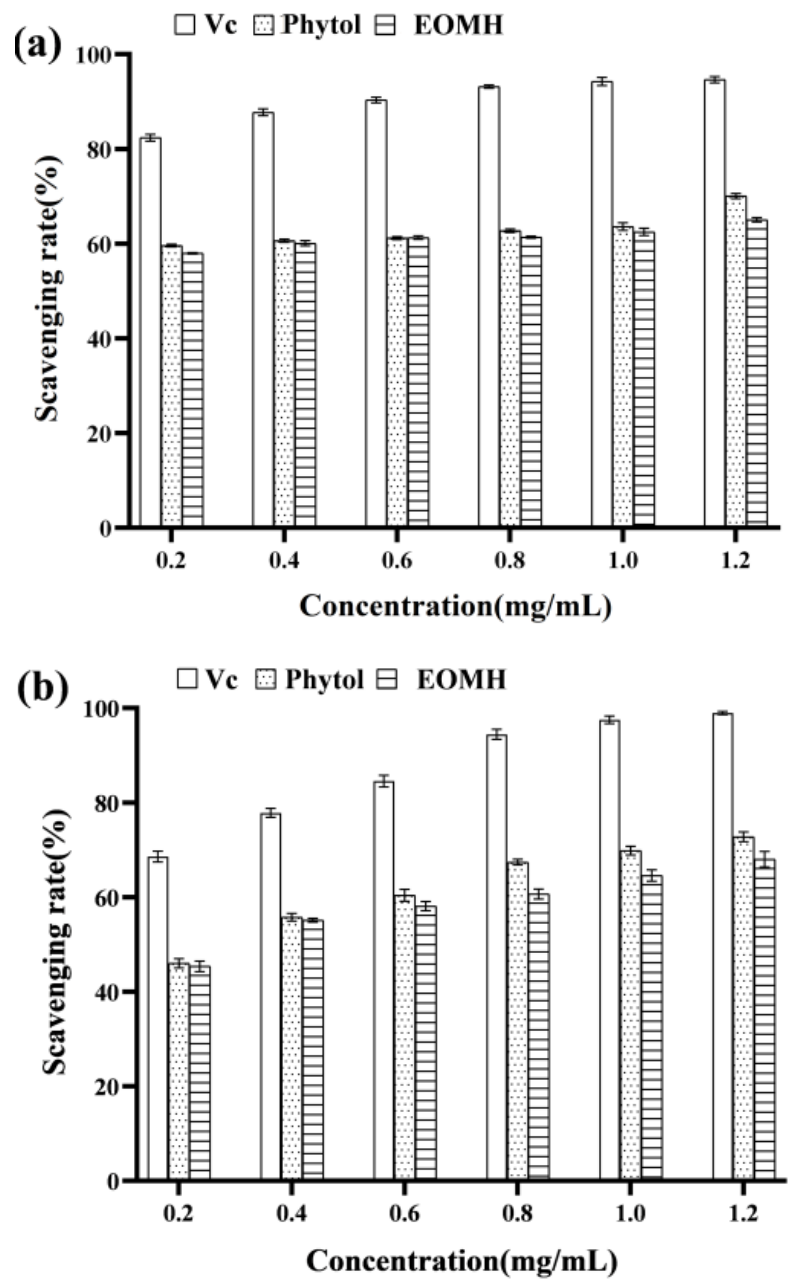

Fig. 6: The in vitro antioxidant activities of EOMH and phytol using $\mathrm{Vc}$ as positive control. (a) $\mathrm{DPPH}^{\bullet}$ scavenging capabilities; (b) ${ }^{\circ} \mathrm{OH}$ scavenging capabilities. Data was shown as means $\pm \operatorname{SD}(n=3)$

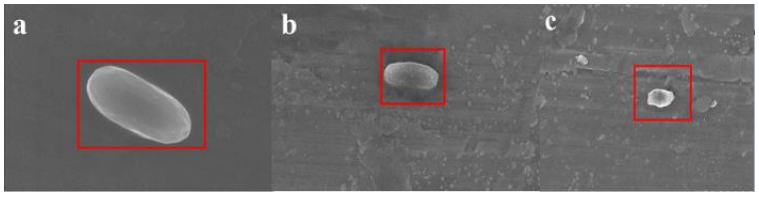

Fig. 7:SEM images of untreated E. coli suspension (a); E. coli suspension treated with phytol (b); E. coli suspension treated with EOMH (c)

\section{Discussion}

In this investigation, the single-factor experiments for EOMH extraction were first conducted for obtaining the independent variables of RSM optimization. From Figs. 1 to 3 , we can observe that extended extraction time and increased particle size as well as excessive liquid-to-solid ratio resulted in a sharp decline in EOMH extraction yield. These phenomena could be uncovered by the following explanations: (1) If the extraction time was too short, due to insufficient heating, the internal structures of materials were not fully destroyed, which largely decreased the release of targeting essential oil. When the materials were sufficiently heated, the release of targeting essential oil was promoted and EOMH yield was largely raised. However, when heating was excessive, the essential oil may flow back with steam, leading to partial consumption and decreasing the extraction rate (Zhang et al., 2014b). (2) If the particle size of dried $M$. hispida was too low, due to the excessively large gaps between materials, heat from steam is easy to escape from the gaps, leading to insufficient heating and restricted the release of essential oil from materials. Generally, to decrease particle size of materials is beneficial for enlarging the surface area, facilitating the even penetrate of heat from steam in materials (Zheljazkov et al., 2014). However, since the extraction was conducted in heterogeneous phase, the materials with too small particle size were apt to be highly dispersed in water, hindering the diffusion of steam, thereby lowering the extraction rate of EOMH (Kusuma and Mahfud, 2016). (3) At low liquid-to-solid ratio, the materials were excessive and cannot be evenly mixed with water (Pourmortazavi and Hajimirsadeghi, 2007). As the liquid-to-solid ratio increased, the materials was in full contact with water to weaken the mass transfer resistance during heating (Najafian, 2013), which can improve the extraction of essential oil. When the liquidto-solid ratio increased further, the evaporated compounds were apt to be redissolved in the abundant water, which may decrease the yield of essential oil (Milojević et al., 2008).

Then, to optimize EOMH extraction, a three-levelthree-factor RSM was designed by BBD using EOMH extraction rate as response value. In previous work, we found that the non-optimized extraction rate of essential 
oil from fresh M. hispida was $0.27 \%$ (Zheng et al., 2019). In this study, considering prolonging the preservation time of $M$. hispida and facilitating the continuous industrial production, we took dried $M$. hispida as raw material to optimize the extraction process of essential oil. The results indicated that although drying may cause the loss of some low-boiling compounds, after process optimization, the loss can be remedied to some extent, making the extraction rate of essential oil from dried $M$. hispida reach $0.31 \%$, which was still higher than that from fresh M. hispida $(0.27 \%)$.

After that, GC-MS was applied to reveal the chemical profile of EOMH (Table 4). Compared with EOFMH (Zheng et al., 2019), there were eight compounds missing in EOMH, considering that these volatile compounds were lost during the drying process of fresh M. hispida (Tang et al., 2014). Amazingly, the content of phytol in EOMH was raised to $53.6 \%$, which was $9.84 \%$ higher than that in EOFMH, suggesting that drying at $60^{\circ} \mathrm{C}$ could be a beneficial processing for the accumulation of phytol in EOMH. Due to the fact that phytol is a famous diterpenoid that possesses prominent bioactivities, particularly antibacterial capacity (Jeong, 2018; de Santos et al., 2013), thus present process would provide a suitable way to obtain phytol-rich essential oil from dried $M$. hispida.

The chemical profile of EOMH was further confirmed by FT-IR analysis by using phytol as a control. The FTIR spectrum comparison of EOMH and phytol (Fig. 5) suggested that EOMH appears to be dominated by phytol. This finding was highly consistent with that of GC-MS analysis (Table 4).

It is deduced from our previous work that the antioxidant ability of phytol may partially contribute to the inhibitory effect of EOMH against E. coli (Zheng et al., 2019). Thus, in present work, the antioxidant abilities of EOMH and phytol were further compared.

Hydroxyl radical is the most active reactive oxygen species. It can react with almost any molecules in living cells (Hou et al., 2020). While, DPPH radical (DPPH') is a very stable nitrogen-containing free radical. If the tested substance can quench $\mathrm{DPPH}^{\circ}$, which may indicate that the tested substance possesses high scanvenging capacities against other unstable radicals such as hydroxyl, alkyl and peroxide free radicals (Zheng et al., 2015). Therefore, in this study, the antioxidant potentials of EOMH were assessed using these two radical models.

As shown in Fig. 6, EOMH possessed similar scavenging capacities against DPPH and hydroxyl radicals with those of phytol, indicating that the antioxidant ability of EOMH could be mainly contributed by phytol.
Finally, the preliminary inhibitory mechanism of EOMH on E. coli was further disclosed via SEM observation. As shown in Fig. 7, these changes on E. coli surface morphology revealed that EOMH would elicit little higher antibacterial capacities against E. coli than that of phytol, suggesting that other factors in addition to the antioxidant mechanism would affect the inhibitory effects of EOMH against E. coli. Reportedly, the esters and alkanes of essential oils can collaboratively exert bacteriostatic effects via degrading and destroying the cell walls and membranes of E. coli, leading to intracellular leakage even cell death (Zhan et al., 2014a). Further research is undergoing.

\section{Conclusion}

Based on preliminary results obtained from our previous work (Zheng et al., 2019), the extraction optimization, constituent identification, in vitro antioxidant capacity measurement and preliminary inhibitory mechanism against $E$. coli of Essential oil from dried $M$. hispida (EOMH) were conducted. On the basis of single-factor tests, EOMH extraction was optimized by RSM and BBD to give the optimal conditions: Extraction time of $7 \mathrm{~h}$, particle size of 20 mesh and liquid-to-solid ratio of 30: $1(\mathrm{~mL} / \mathrm{g})$, under which the highest extraction rate was found to be $0.31 \%$. Nineteen components were identified in EOMH by GC-MS and phytol content accounted for $53.6 \%$, which was $9.84 \%$ higher than that in the Essential oil from Fresh M. hispida (EOFMH) (Zheng et al., 2019). Infrared spectroscopy showed that EOMH has characteristic absorption peaks similar to those of phytol, which further validated the results that EOMH is dominated by phytol (Table 4). Antioxidant evaluation showed that the antioxidant ability of EOMH could be mainly contributed by the relatively higher proportion of phytol. SEM analysis demonstrated that EOMH is more destructive to E. coli than that of phytol. It was deduced that other factors would equally affect the inhibitory effects of EOMH against E. coli besides the antioxidant mechanism. Hence, two issues are planned to be further studied in the future: (1) To further explain the antibacterial mechanism of EOMH against Escherichia coli by Transmission Electron Microscopy (TEM), cell membrane permeability measurement and the effects on the physiological metabolism; (2) To develop novel EOMH-based antioxidants and bacteriostatic agents by using $M$. hispida as raw materials.

Finally, this study is expected to offer a scientific basis for high-valued utilization of $M$. hispida species.

\section{Funding Information}

This work was supported by the innovation training program and the key graduation thesis program for undergraduate students of Changshu Institute of Technology. 


\section{Author's Contributions}

Xiulan Chu and Tong Wang: Performed the experiments of extraction and antioxidant capacity.

Qianfeng Chen, Xinyu Bai and Chen Pan: Carried out FT-IR analysis and bacteriostatic experiments.

Yang Zhang: Designed the experiments, revised the manuscript and polish the manuscript.

Lixue Zheng: Designed the experiments, wrote the manuscript and polish the language.

\section{Ethics}

This article is original and contains unpublished material. The corresponding author confirms that all of the other authors have read and approved the manuscript and no ethical issues involved.

\section{References}

Boughendjioua, H., Mezedjeri, N. \& Idjouadiene, I. (2020). Chemical constituents of Algerian mandarin (Citrus reticulata) essential oil by GC-MS and FT-IR analysis. Current Issues in Pharmacy and Medical Sciences, 33(4), 197-201. doi.org/10.2478/cipms-2020-0032

Cassel, E., Vargas, R. M. F., Martinez, N., Lorenzo, D., \& Dellacassa, E. (2009). Steam distillation modeling for essential oil extraction process. Industrial Crops and Products, 29(1), 171-176. doi.org/10.1016/j.indcrop.2008.04.017

Chen, H., \& Yen, G. (2007). Antioxidant activity and free radical-scavenging capacity of extracts from guava (Psidium guajava L.) leaves. Food chemistry, 101(2),686-694.

doi.org/10.1016/j.foodchem.2006.02.047

Cheng, J., Xiang, M., Wu, T., Zhang, Y. Y., Peng, C. C., Lei, L., \& Song, H. P. (2016). Effects of TSMP on glucose and fat metabolism as well as functions of pancreatic islet cells in type 2 diabetic rats. Chinese Journal of Hospital Pharmacy, 36(019), 1625-1628.

doi.org/10.13286/j.cnki.chinhosppharmacyj.2016. 19.02

Cui, H., Pan, H. W., Wang, P. H., Yang, X. D., Zhai, W. C., Dong, Y., \& Zhou H. L. (2018). Essential oils from carex meyeriana Kunth: Optimization of hydrodistillation extraction by response surface methodology and evaluation of its antioxidant and antimicrobial activities. Industrial Crops \& Products, 124(2018), 669-676. doi.org/10.1016/j.indcrop.2018.08.041

Elkady, W. M., \& Ayoub, I. M. (2018). Chemical profiling and antiproliferative effect of essential oils of two Araucaria species cultivated in Egypt. Industrial Crops and Products, 118(2018), 188-195. doi.org/10.1016/j.indcrop.2018.03.051
Guo, Y., Zheng, H., Zhang, H., Ma, L., Han, J., \& Li, K. (2012). Optimization of combined microwaveultrasonic wave extraction of cochineal dye by response surface methodology. Applied Mechanics and Materials, 161(2012), 82-87. doi.org/10.4028/www.scientific.net/AMM.161.8

Hou, J. T., Zhang, M., Liu, Y., Ma, X., Duan, R., Cao, X., \& Ren, W. X. (2020). Fluorescent detectors for hydroxyl radical and their applications in bioimaging: A review. Coordination Chemistry Reviews, 421, 213457. doi.org/org/10.1016/j.ccr.2020.213457

Huang, H., Wang, H., Yih, K., Chang, L., \& Chang, T. (2012). Dual bioactivities of essential oil extracted from the leaves of Artemisia argyi as an antimelanogenic versus antioxidant agent and chemical composition analysis by GC/MS. International Journal of Molecular Sciences, 13(12), 14679-14697. doi.org/10.3390/ijms 131114679

Huang, X. J., Nie, S. P., Wang, Y. T., Wang, Y. W., Cai, H. L., Tian, F., \& Cui, W. W. (2013) Optimized extraction and compositional analysis of polysaccharides from dried stems of Dendrobium officinale. Food Science, 34(22), 21-26. doi.org/10.7506/spkx1002-6630-201322005

Javad, S., Abdolrasoul, H.E., Zahra, D., \& Hossein, B. (2009). GC/MS analysis and in vitro antioxidant activity of essential oil and methanol extracts of Thymus caramanicus Jalas and its main constituent carvacrol. Food Chemistry, 115(4), 1524-1528. doi.org/10.1016/j.foodchem.2009.01.051

Jeong, S.H. (2018). Inhibitory effect of phytol on cellular senescence. Biomedical Dermatology, 2, 13. doi.org/10.1186/s41702-018-0025-8

Krakowska, A., Rafińska, K., Walczak, J., Kowalkowski, T., \& Buszewski, B. (2017). Comparison of various extraction techniques of Medicago sativa: Yield, antioxidant activity and content of phytochemical constituents. Journal of AOAC International, 100(6), 1681-1693. doi.org/10.5740/jaoacint.17-0234

Kusuma, H.S., \& Mahfud, M. (2016). Comparison of conventional and microwave-assisted distillation of essential oil from Pogostemon cablin leaves: Analysis and modeling of heat and mass transfer. Journal of Applied Research on Medicinal and Aromatic Plants, 2016, 55-65. doi.org/10.1016/j.jarmap.2016.08.002

Liu, X.G., Huang, M.Y., Lv, M.C., Sun, Y.Q., Bian, J., Gao, P.Y., \& Li, D.Q. (2018). Research progress on chemical composition and biological activities of Medicago sativa L. Science and Technology of Food Industry, 39(11), 344-352. doi.org/10.13386/j.issn1002-0306.2018.11.059

Milojević, S.Ž., Stojanović, T.D., Palić, R., Lazić, M. L., \& Veljković, V.B. (2008). Kinetics of distillation of essential oil from comminuted ripe juniper (Juniperus communis L.) berries. Biochemical Engineering Journal, 39(3), 547-553. doi.org/10.1016/j.bej.2007.10.017 
Mirza, M., \& Navaei, M.N. (2009). Chemical composition of the essential oils extracted from the leaf and flowers of Marsdenia erecta (L.) R. Br. in Iran. Journal of Essential Oil Bearing Plants, 12(1), 87-91. doi.org/10.1080/0972060x.2009.10643697

Najafian, S. (2013). Rapid extraction and analysis of volatile oils components of Melissa officinalis using headspace and Gas Chromatography/Mass Spectrometry. Journal of Herbs, Spices \& Medicinal Plants, 19(4), 340-347. doi.org/10.1080/10496475.2013.79910

Pourmortazavi, S.M., \& Hajimirsadeghi, S.S. (2007). Supercritical fluid extraction in plant essential and volatile oil analysis. Journal of Chromatography A, 1163(2007), 2-24. doi.org/10.1016/j.chroma.2007.06.021

Santos, C.C.D.M.P., Salvadori, M.S., Mota, V.G., Costa, L.M., de Almeida, A.A.C., de Oliveira, G.A.L., \& de Almeida, R.N. (2013). Antinociceptive and antioxidant activities of phytol in vivo and in vitro models. Neuroscience Journal, 2013. doi.org/10.1155/2013/949452

Tang, W.W., Li, G.Q., \& Jin, X.J. (2014). Effects of diffierent drying methods on components of volatile oil of angelica sinensis. Chinese Journal of Experimental Traditional Medical Formulae, 20(003), 9-12. doi.org/10.11653/syfj2014030009

Wang, L., Tan, N., Hu, J., Wang, H., Duan, D., Ma, L., Xiao, J., \& Wang, X. (2017). Analysis of the main active ingredients and bioactivities of essential oil from Osmanthus fragrans Var. thunbergii using a complex network approach. BMC Systems Biology, 11(1), 144-155. doi.org/10.1186/s12918-017-0523-0
Wang, X.X., Chen, L.L., Zhang, Y.W., \& Mao, P.S. (2016). Determination of hard rate of alfalfa (Medicago sativa L.) seeds with near infrared spectroscopy. Spectroscopy and Spectral Analysis, 36(3), 702-705. doi.org/10.3964/j.issn.1000-0593(2016)03-0702-04

Zhan, G., Pan, L.Q., Mao, S.B., Zhang, W., Wei, Y.Y., \& Tu, K. (2014a). Study on antibacterial properties and major bioactive constituents of Chinese water chestnut (Eleocharis dulcis) peels extracts/fractions. European Food Research and Technology, 238(5), 789-796. doi.org/10.1007/s00217-013-2151-2

Zhang, L., Xue, Z., Ni, L., \& Ma, D. (2014b). Extraction and quality analysis of volatile oils from onions by coupling pilot and laboratory equipment based on multi-rectification. Separation and Purification Technology, 137(2014), 36-42. doi.org/10.1016/j.seppur.2014.09.022

Zheljazkov, V.D., Astatkie, T., \& Schlegel, V. (2014). Hydrodistillation extraction time effect on essential oil yield, composition and bioactivity of coriander oil. Journal of Oleo Science, 63(9), 857-865. doi.org/10.5650/jos.ess 14014

Zheng, L., Ben, L., Cui, Z., Fu, Q., Wang, L., Qi, B., \& Zhang, Y. (2019). The phytol-rich essential oil from fresh Medicago hispida exerts significant inhibitory activity against Escherichia coli. American Journal of Biochemistry and Biotechnology, 15(4), 270-274. doi.org/10.3844/ajbbsp.2019.270.274

Zheng, L., Lin, L., Su, G., Zhao, Q., \& Zhao, M. (2015). Pitfalls of using 1, 1-diphenyl-2picrylhydrazyl (DPPH) assay to assess the radical scavenging activity of peptides: Its susceptibility to interference and low reactivity towards peptides. Food Research International, 76, 359-365. doi.org/10.1016/j.foodres.2015.06.045 